Europhys. Lett., 74 (2), pp. 195-201 (2006)

DOI: $10.1209 / \mathrm{epl} / \mathrm{i} 2006-10007-\mathrm{y}$

\title{
Free-energy barriers in the Sherrington-Kirkpatrick model
}

\author{
E. BitTneR and W. JANKE \\ Institut für Theoretische Physik and Centre for Theoretical Sciences (NTZ) \\ Universität Leipzig - Augustusplatz 10/11, D-04109 Leipzig, Germany
}

received 20 January 2005; accepted in final form 27 February 2006

published online 24 March 2006

PACS. 02.70.Uu - Applications of Monte Carlo methods.

PACS. 75.10.Nr - Spin-glass and other random models.

\begin{abstract}
The Sherrington-Kirkpatrick spin-glass model is investigated by means of Monte Carlo simulations by employing a combination of the multi-overlap algorithm with parallel tempering methods. We investigate the finite-size scaling behaviour of the free-energy barriers which are visible in the probability density of the Parisi overlap parameter. Assuming that the mean barrier height diverges with the number of spins $N$ as $N^{\alpha}$, our data show good agreement with the theoretical value $\alpha=1 / 3$.
\end{abstract}

Despite three decades of research the nature of the "glassy" low-temperature phase of finite-dimensional spin-glass systems remains a major open problem in statistical physics. It is still unresolved whether the replica symmetry-breaking theory or the phenomenological droplet picture yields the correct description (for reviews, see refs. [1-4]). Even at the meanfield level, only very recently a mathematical proof [5] of Parisi's replica solution [6] for the Sherrington-Kirkpatrick (SK) model [7] was given.

In the thermodynamic limit the frozen phase of the mean-field spin glass shows many stable and metastable states. Such a feature is the consequence of the disorder and the frustration characterizing spin glasses in general and leading to a rugged free-energy landscape with probable regions (low free energy) separated by rare-event states (high free energy). But also for finite systems the free-energy landscape shows an intricate, corrugated structure. Therefore, it is hard to measure the free-energy barriers by means of conventional Monte Carlo simulations directly. The aim of this letter is to study the free-energy barriers of the SK mean-field spin-glass model using the multi-overlap Monte Carlo algorithm [8] and to compare our results with previous findings for finite-range spin glasses [9]. For analyzing the barriers, we used precisely the same method as introduced in ref. [9] for the Edwards-Anderson (EA) nearest-neighbor model [10], where the scaling of the mean barrier height with the number of spins was found to clearly deviate from the theoretical mean-field prediction in both three and four dimensions. To exclude the possibility that this deviation could, in principle, be caused by employing different definitions in the theoretical and computational work, it is important to apply precisely the same numerical procedure to the SK model.

The Hamiltonian of the SK model reads

$$
H=-\sum_{i<j} J_{i j} s_{i} s_{j}
$$


where $s_{i}= \pm 1, i=1, \ldots, N$, and the $J_{i j}$ are independent random variables with a Gaussian distribution of zero mean and variance $N^{-1}, N$ being the numbers of spins. The critical temperature of the infinite system is $T_{c}=1$.

The fact that there is no parametrization of the relevant configurations by a conventional thermodynamic variable led us to use the Parisi overlap parameter [6],

$$
q=\frac{1}{N} \sum_{i=1}^{N} s_{i}^{(1)} s_{i}^{(2)}
$$

as an order parameter, where the spin superscripts label two independent (real) replicas for the same realization of randomly chosen exchange coupling constants $\mathcal{J}=J_{i j}$. For given $\mathcal{J}$ the probability density of $q$ is denoted by $P_{\mathcal{J}}(q)$, and the function $P(q)$ is obtained as

$$
P(q)=\left[P_{\mathcal{J}}(q)\right]_{\mathrm{av}}=\frac{1}{\# J} \sum_{\mathcal{J}} P_{\mathcal{J}}(q),
$$

where $\# J$ is the number of realizations considered. For a given realization of $\mathcal{J}$ the nontrivial (i.e., away from $q= \pm 1$ ) minima are related to the free-energy barriers of this disordered system $\mathcal{J}$. We are, therefore, interested in the whole range of the probability density $P_{\mathcal{J}}(q)$. Conventional, canonical Monte Carlo simulations are not suited for such systems because the likelihood to generate the corresponding rare-event configurations in the Gibbs canonical ensemble is very small. This is overcome by non-Boltzmann sampling $[11,12]$ with the multioverlap weight [8]

$$
w_{\mathcal{J}}(q)=\exp \left[\beta \sum_{i<j} J_{i j}\left(s_{i}^{(1)} s_{j}^{(1)}+s_{i}^{(2)} s_{j}^{(2)}\right)+S_{\mathcal{J}}(q)\right],
$$

where the two replicas are coupled by $S_{\mathcal{J}}$ in such a way that a broad multi-overlap histogram $P_{\mathcal{J}}^{\text {muq }}(q)$ over the entire accessible range $-1 \leq q \leq 1$ is obtained. When simulating with the multi-overlap weight, canonical expectation values of any quantity $\mathcal{O}$ can be reconstructed by reweighting,

$$
\langle\mathcal{O}\rangle_{\mathcal{J}}^{\text {can }}=\left\langle\mathcal{O} \exp \left[-S_{\mathcal{J}}\right]\right\rangle_{\mathcal{J}} /\left\langle\exp \left[-S_{\mathcal{J}}\right]\right\rangle_{\mathcal{J}}
$$

Ideally, the weight function $W_{\mathcal{J}} \equiv \exp \left[S_{\mathcal{J}}\right]$ should satisfy

$$
P_{\mathcal{J}}^{\text {muq }}(q)=P_{\mathcal{J}}^{\text {can }} W_{\mathcal{J}}=\text { const }
$$

i.e., it should give rise to a completely flat multi-overlap probability density $P_{\mathcal{J}}^{\text {muq }}(q)$. Of course, $P_{\mathcal{J}}(q)$ is a priori unknown and one has to proceed by iteration. An efficient way to construct the weight function $W_{\mathcal{J}}$ is to use an accumulative recursion, in which the new weight factor is computed from all available data accumulated so far; for details see refs. [13] and [14]. The multi-overlap algorithm combined with this recursion allows an almost automatic simulation of the SK model.

Let us now turn to the description of the Monte Carlo update procedure used by us. We combined the multi-overlap algorithm [8] as described above with the parallel tempering optimized Monte Carlo procedure [15] to overcome as many as possible hidden barriers in the rugged phase space. We studied systems with $N=32,64,128,256,512$ and 1024 spins. For the parallel tempering procedure we chose a set of 32 temperature values in the range $T=1 / 3^{-}$ 1.6 for all of our systems apart from the largest, where we used 64 temperature values for the 
same temperature interval. Once the entire range of $q$ for all temperature values was covered, the accumulative recursion for the weight functions was stopped. Due to large differences in the free-energy landscape for different disorder realizations $\mathcal{J}$, the number of recursion steps varied for different $\mathcal{J}$. After the weight functions were constructed, they were kept fixed and we took about 100000 measurements, with five sweeps between the measurements. A sweep consisted of $N$ spin flips with the multi-overlap algorithm and one parallel tempering update. We recorded time series of the overlap parameter $q$ for five different temperature values and the canonical $P_{\mathcal{J}}(q)$ distribution for all temperature values. To average over the disorder we used 1000 realizations of the disorder for $N \leq 512$ and 100 for $N=1024$.

For each of these samples $\mathcal{J}$ we computed the barrier autocorrelation time $\tau_{B}$ by employing the same method as Berg et al. used for the $\pm J$ EA Ising spin-glass model [9]. For clarity, we recall the basic idea here. The free-energy barrier $F_{B}$ for a given $P_{\mathcal{J}}(q)$ is defined through the autocorrelation time of a one-dimensional Markov process which has the canonical $P_{\mathcal{J}}(q)$ distribution as equilibrium state. The transition probabilities $T_{i, j}$ are given by

$$
T=\left[\begin{array}{cccc}
1-w_{2,1} & w_{1,2} & 0 & \cdots \\
w_{2,1} & 1-w_{1,2}-w_{3,2} & w_{2,3} & \cdots \\
0 & w_{3,2} & 1-w_{2,3}-w_{4,3} & \cdots \\
0 & 0 & w_{4,3} & \cdots \\
\vdots & \vdots & \vdots & \ddots
\end{array}\right],
$$

where $w_{i, j}(i \neq j)$ is a probability $\grave{a}$ la Metropolis to jump from state $q=q_{j}$ to $q=q_{i}$ $\left(q_{i}=i / N, i \in[-N,-N+2, \ldots,+N]\right)$,

$$
w_{i, j}=\frac{1}{2} \min \left(1, \frac{P_{\mathcal{J}}\left(q_{i}\right)}{P_{\mathcal{J}}\left(q_{j}\right)}\right) .
$$

The transition matrix $T$ fulfils the detailed balance condition (with $P_{\mathcal{J}}$ ), and as a consequence it has only real eigenvalues. The largest eigenvalue (equal to one) is non-degenerate, and the second largest eigenvalue $\lambda_{1}$ determines the autocorrelation time of the Markov chain,

$$
\tau_{B, \mathcal{J}}=\frac{1}{N\left(1-\lambda_{1}\right)} .
$$

The associated free-energy barrier for realization $\mathcal{J}$ is defined as

$$
F_{B, \mathcal{J}}=\ln \left(\tau_{B, \mathcal{J}}\right)
$$

Note that the definition of the autocorrelation time (9) takes only barriers in $q$ into account, but not other barriers which may well exist in the multidimensional configuration space.

For each temperature value we performed least-squares fits of the finite-size scaling (FSS) ansatz $F_{B} \equiv\left[F_{B, \mathcal{J}}\right]_{\mathrm{av}}=c N^{\alpha}$ which corresponds to the exponential FSS behaviour

$$
\tau_{B} \propto e^{c N^{\alpha}} .
$$

The results from these fits are depicted in fig. 1 and are consistent with previous results in the literature [16-23] using numerical and analytical methods. The horizontal line in fig. 1 indicates the theoretical value $\alpha=1 / 3$ of ref. [21]. The three data sets show fits with different lower bounds of the fit range $N_{\mathrm{min}}$, while the upper bound was always our largest system $N=1024$. From these fits we see a strong finite-size effect for $T \rightarrow T_{c}=1$. At lower 


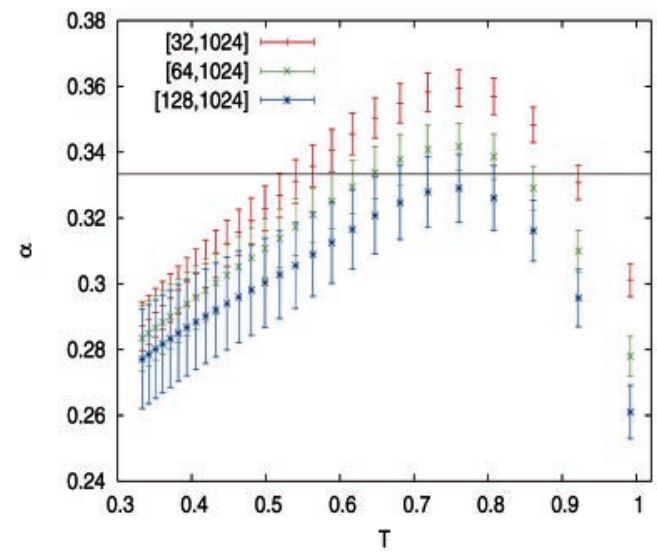

Fig. 1 - Dependence of the exponent $\alpha$ in the ansatz $F_{B}=c N^{\alpha}$ on the lower bound of the fit range $\left[N_{\min }, 1024\right]$ as a function of temperature. The horizontal line indicates the theoretical value $\alpha=1 / 3$.

temperatures we find a linearly increasing deviation from the theoretical value. This is also a finite-size effect, because the slope of the deviation becomes flatter when increasing the lower bound of the fit range and there is no physical reason for a change of behaviour of the barrier autocorrelation time in the glassy phase.

One possible explanation for this deviation from the theoretical value is the lack of selfaveraging of the finite-volume Parisi overlap parameter distribution $P_{\mathcal{J}}$ in the SK model [24]. To check the non-self-averaging of our numerical data, we analyzed the free-energy barriers relying on the (empirical) cumulative distribution function $D(x)$, which is defined for a set of sorted data, e.g., the free-energy barriers $F_{B, 1}<F_{B, 2}<\ldots<F_{B, n}$, by

$$
\frac{i}{n}-\frac{1}{2 n} \leq D(x) \leq \frac{i}{n}+\frac{1}{2 n} \quad \text { for } \quad F_{B, i} \leq x \leq F_{B, i+1}
$$

where we use a straight-line interpolation in between. A nice way to test the non-self-averaging is to look at the peaked distribution function [9]:

$$
D_{Q}(x)= \begin{cases}D(x) & \text { for } D(x) \leq 0.5 \\ 1-D(x) & \text { for } D(x) \geq 0.5\end{cases}
$$

This function has a peak at the median $x_{\text {med }}$ of the data with $D_{Q}=0.5$. For self-averaging data $x$, the function $D_{Q}$ collapses in infinite volume to $D_{Q}(x)=0.5$ for $x=\bar{x}$ and 0 otherwise, where $\bar{x}$ is the mean value. For non-self-averaging quantities the width of $D_{Q}$ stays finite. In fig. 2 we show the peaked distribution function in units of the median value which also scales approximately with $N^{1 / 3}$. We observe a very weak finite-size dependence and therefore clearly a non-self-averaging behaviour of the free-energy barriers. This result is in agreement with theoretical calculations [24]. The width of $D_{Q}$ increases with decreasing temperature, i.e., the sample to sample variations become more pronounced for lower temperatures.

We already mentioned that the distribution of the free-energy barriers becomes broader for low temperatures. Now let us have a closer look at the distribution itself, therefore we only analyze the system sizes where we have 1000 different disorder realizations. In recent work, Dayal et al. [25] have found that the tunnelling times of their flat-histogram sampling simulations of the $2 \mathrm{D} \pm J$ EA Ising spin glass are distributed according to the Fréchet extremal value distribution for fat-tailed distributions. In general, extreme-value statistics can 


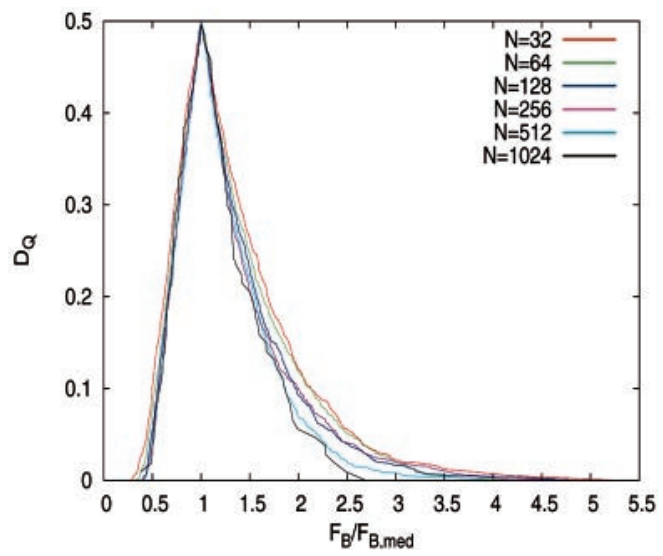

Fig. 2 - Probability distribution function $D_{Q}$ for the free-energy barriers at $T=1 / 3$ in units of their median value.

be classified into different universality classes [26,27], depending on whether the tails of the original distribution are fat-tailed (algebraic), exponential, or thin-tailed (decaying faster than exponential). Assuming that the free-energy barriers of the SK model are, as well as the tunnelling times of the EA spin glass, distributed according to an extreme-value distribution, we use the integrated probability density of the generalized extreme value distribution (GEV),

$$
F_{\xi ; \mu ; \sigma}(x)=\exp \left[-\left(1+\xi \frac{x-\mu}{\sigma}\right)^{-1 / \xi}\right]
$$

for $1+\xi(x-\mu) / \sigma>0$, to fit our data. Here, $\xi$ is the shape parameter, and $\mu$ and $\sigma$ are related to the $N$-dependent mean and variance of the distribution, respectively. We find that the free-energy barriers show fat tails for $T<T_{c}$ with shape parameter $\xi>0$, i.e., a Fréchet distribution. In fig. 3 we plot the resulting fits and data of the probability density for different temperatures below the glass transition and find that the tails become fatter and fatter as the temperature goes to zero. The histograms for low temperatures show deviations from the

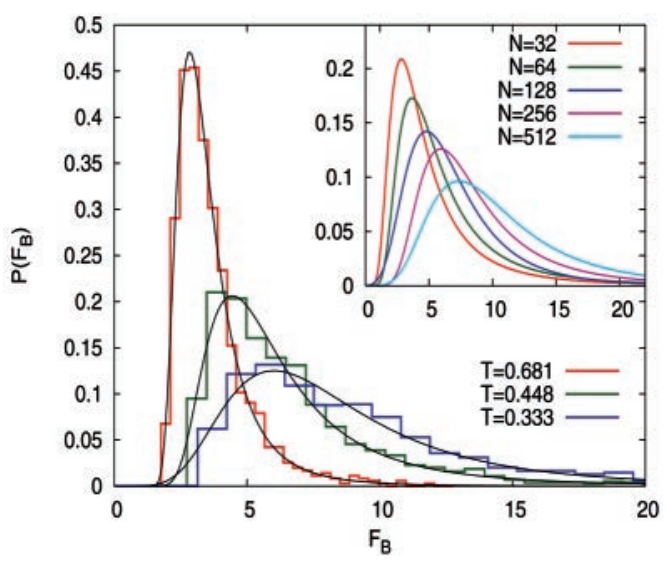

Fig. 3 - Density of free-energy barriers $F_{B}$ for $N=256$ at different temperatures. The inset shows the densities for $T=1 / 3$ for different numbers of spins. 


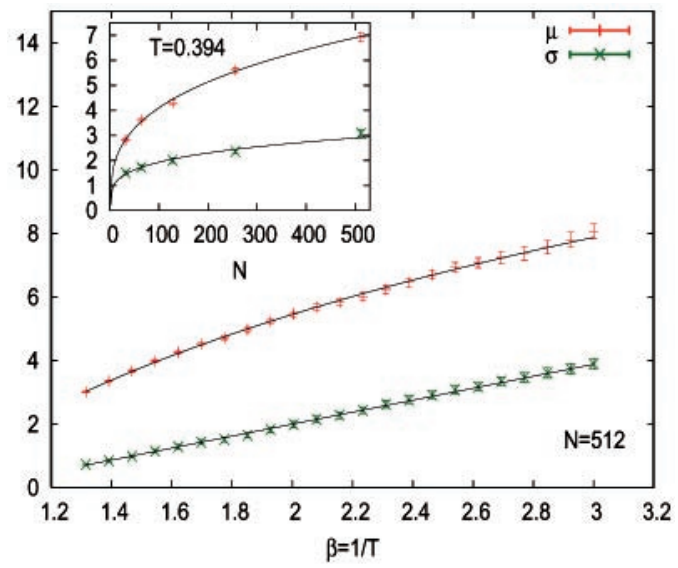

Fig. 4 - Temperature dependence of the parameters $\mu$ and $\sigma$ of the Fréchet distribution for $N=512$. The inset shows the size dependence of $\mu$ and $\sigma$ for $T=0.394$.

Fréchet distribution for small values of $F_{B}$, so a much larger number of disorder realizations would be needed to determine both tails of the distribution properly. We determined the parameters $\mu, \sigma$ and $\xi$ for different temperatures and found that $\mu$ grows logarithmically and $\sigma$ linearly with inverse temperature $1 / T$, whereas $\xi$ stays more or less constant at $\xi \approx 0.33$. As an example, we show in fig. 4 the results for $N=512$. If we keep the temperature fixed and look at the size dependence of the distribution, we find that for a larger number of spins the distribution becomes broader, cf. the inset of fig. 3. To quantify this behaviour we use the scaling relations $\mu \propto N^{\alpha_{\mu}}$ and $\sigma \propto N^{\alpha_{\sigma}}$, which lead to $\alpha_{\mu} \approx 0.31$ and $\alpha_{\sigma} \approx 0.25$ for our lowest temperatures, see the inset of fig. 4 . The exponent of the location parameter $\mu$ is closely related to the scaling exponent of the mean barrier height, therefore $\alpha_{\mu}$ should be $1 / 3$, which is in good agreement with our result. We find a weak temperature dependence of the exponents $\alpha_{\mu}$ and $\alpha_{\sigma}$ with positive and negative slope for increasing $T$, respectively.

As a short remark, we want to mention that we also looked at the correlation between the $n$ largest eigenvalues $\lambda_{n}$ of the interaction matrix $J_{i j}$, which is a real symmetric matrix, and the largest free-energy barrier $F_{B}$ for this disorder realisation. And we find for $T<T_{c}$ a very weak but non-vanishing correlation between the largest eigenvalue $\lambda_{1}$ and $F_{B}$. Unfortunately, this correlation becomes weaker and weaker for larger systems and also for smaller temperatures. All other correlations vanish much faster, such that these correlations cannot be used to predict the magnitude of the free-energy barrier based only on $J_{i j}$.

To conclude, we found that the free-energy barriers of the SK model are non-self-averaging and distributed according to the Fréchet extremal value distribution. These particular features were also found for the EA nearest-neighbor model and such similarities support the position that the Parisi replica symmetry-breaking solution of the SK model is the limit of the shortrange model on a lattice in dimension $d$ when $d \rightarrow \infty$, with a proper rescaling of the strength of the Hamiltonian. On the other hand, we also found that the free-energy barriers diverge with the theoretically predicted value $\alpha=1 / 3$, which is in contrast to the results for the EA model in three and four dimensions [9]. Of course, one reason for this discrepancy could possibly be that finite-size effects, caused by the relative large temperature used in ref. [9], are too strong or the lattice sizes are too small to see the real asymptotic behaviour. Still, our results also support the complementary position, that the Parisi replica symmetry-breaking solution sheds little light on the thermodynamic structure of the EA model [28]. With these 
oppositional results we are not able to rule out one of the two scenarios. But we can state that the method to determine the free-energy barriers proposed by Berg et al. [9] leads to correct results for the SK model and is very useful for systems with many barriers in the free energy.

We gratefully acknowledge financial support from the Deutsche Forschungsgemeinschaft (DFG) under Grant No. JA 483/22-1 and the EU RTN-Network "ENRAGE": Random Geometry and Random Matrices: From Quantum Gravity to Econophysics under grant No. MRTNCT-2004-005616.

\section{REFERENCES}

[1] Young A. P. (Editor), Spin Glasses and Random Fields (World Scientific, Singapore) 1997.

[2] Fischer K. H. and Hertz J. A., Spin Glasses (Cambridge University Press, Cambridge, England) 1991.

[3] Mezard M., Parisi G. and Virasoro M. A., Spin Glass Theory and Beyond (World Scientific, Singapore) 1987.

[4] Binder K. and Young A. P., Rev. Mod. Phys., 58 (1986) 801.

[5] Talagrand M., C. R. Acad. Sci. Paris, Ser. I, 337 (2003) 111.

[6] PARisi G., Phys. Rev. Lett., 43 (1979) 1754.

[7] Sherrington D. and Kirkpatrick S., Phys. Rev. Lett., 35 (1975) 1792.

[8] Berg B. A. and Janke W., Phys. Rev. Lett., 80 (1998) 4771.

[9] Berg B. A., Billoire A. and Janke W., Phys. Rev. B, 61 (2000) 12143.

[10] Edwards S. F. and Anderson P. W., J. Phys. F, 5 (1975) 965.

[11] Berg B. A., Fields Inst. Commun., 26 (2000) 1.

[12] Janke W., Physica A, 254 (1998) 164.

[13] Berg B. A., J. Stat. Phys., 82 (1996) 323.

[14] Janke W., Histograms and All That, in Computer Simulations of Surfaces and Interfaces, Proceedings of the NATO Advanced Study Institute, Albena, Bulgaria, September 2002, edited by Dünweg B., Landau D. P. and Milchev A. I., NATO Sci. Ser., II, Math., Phys. Chem., Vol. 114 (Kluwer, Dordrecht) 2003, p. 137.

[15] Hukushima K. and Nemoto K., J. Phys. Soc. Jpn., 65 (1996) 1604.

[16] Mackenzie N. D. and Young A. P., Phys. Rev. Lett., 49 (1982) 301; J. Phys. C, 16 (1983) 5321.

[17] Nemoto K., J. Phys. A, 21 (1988) L287.

[18] Rodgers G. J. and Moore M. A., J. Phys. A, 22 (1989) 1085.

[19] Vertechi D. and Virasoro M. A., J. Phys. (Paris), 50 (1989) 2325; Europhys. Lett., 12 (1990) 589.

[20] Colborne S. G. W., J. Phys. A, 23 (1990) 4013.

[21] Kinzelbach H. and Horner H., Z. Phys. B, 84 (1991) 95.

[22] Takayama H., Yoshino H. and Hukushima K., J. Phys. A, 30 (1997) 3891.

[23] Billoire A. and Marinari E., J. Phys. A, 34 (2001) L727.

[24] Pastur L. A. and Shcherbina M. V., J. Stat. Phys., 62 (1992) 1.

[25] Dayal P., Trebst S., Wessel S., Würtz D., Troyer M., Sabhapandit S. and CopperSmith S. N., Phys. Rev. Lett., 92 (2004) 097201.

[26] Gumbel E. J., Statistics of Extremes (Columbia University Press, New York) 1958.

[27] Galambos J., The Asymptotic Theory of Extreme Order Statistics, 2nd edition (Krieger Publishing, Malibar, Florida) 1987.

[28] Newman C. M. and Stein D. L., Phys. Rev. Lett., 91 (2003) 197205. 